\title{
Pooled analysis of C-Reactive Protein levels and mortality in prostate cancer patients
}

Julie N. Graff ${ }^{1,2}$, Tomasz M. Beer ${ }^{2}$, Bian Liu ${ }^{3}$, Guru Sonpavde ${ }^{4}$, Emanuela Taioli ${ }^{3}$

${ }^{1}$ Portland VA Medical Center, Portland, OR; ${ }^{2}$ Knight Cancer Institute, Oregon Health \& Science University, Portland, OR; ${ }^{3}$ Hofstra North Shore-LIJ School of Medicine; The Feinstein Institute for Medical Research, Manhasset, NY; ${ }^{4}$ University of Alabama at Birmingham (UAB) Comprehensive Cancer Center, Birmingham, AL

\section{Corresponding author:}

Emanuela Taioli MD PhD

Hofstra North Shore-LIJ School of Medicine and

North Shore/LIJ Health System, The Feinstein Institute for Medical Research

175 Community Drive, Rm 203

Great Neck, NY 11021

tel: (516) 4653093 


\section{ABSTRACT}

Introduction: Previous studies have reported that higher CRP levels are significantly associated with worse outcome in prostate cancer patients. The size of each individual study was not large enough to allow sufficient statistical power to draw conclusions. We conducted a pooled analysis of individual data of published studies to evaluate the association between elevated CRP and risk of death in prostate cancer, and to find the best CRP cut-off that could predict mortality.

Methods: Original research studies on prostate cancer survival and CRP levels were identified $(n=6)$. Corresponding authors were contacted and invited to share individual data. Two data sets were received (235 patients). The combined hazard ratio (HR) was calculated and adjusted for age, PSA, Hb, Alkaline Phosphatase. The best cutoff of CRP was explored using X-title software.

Results: High CRP was statistically significant associated with mortality [HRmeta 1.83 (95\% CI: 1.51-2.21)], without evidence of heterogeneity among studies. At pooled analysis, adjusted $\mathrm{HR}_{\text {pooled }}$ for CRP $<5 \mathrm{vs} \geq 5 \mathrm{mg} / \mathrm{l}$ was 1.44 (1.02-20.4). The best CPR cutoff was $12 \mathrm{mg} / \mathrm{l}$ : the adjusted $\mathrm{HR}_{\text {pooled }}$ for $\mathrm{CRP}<12 \mathrm{vs} \geq 12 \mathrm{mg} / \mathrm{l}$ was $1.53(1.01-2.32)$

Conclusions: Elevated CRP levels are associated with overall survival in prostate cancer patients. Since CRP is an affordable and readily available assay, it may hold promise in improving prognostication and potentially to predict the activity of specific therapeutic agents.

Key words: outcome, pooled analysis, systemic inflammation

MICRO ABSTRACT: this is a pooled analysis of studies on C-Reactive Protein (CRP) and prostate cancer mortality. 235 patients are included. CRP was significantly associated with mortality; the best predictor cut-off is CRP $<12 \mathrm{mg} / \mathrm{L}$. CRP is a routine assay that could be tested at diagnosis to improve prognostication of prostate cancer patients.

\section{Clinical Practice Points:}

What is already known about this subject: it is suggested that high C-Reactive Protein (CPR) levels are significantly associated with worse outcome in prostate cancer patients.

What are the new findings: Pooled analysis of 235 prostate cancer patients with complete clinical data shows that CRP is significantly associated with mortality.

The best CPR cutoff to predict mortality was $12 \mathrm{mg} / 1$.

How might it impact on clinical practice in the foreseeable future: CPR, an easy and routinely performed laboratory test, could be considered in clinical practice to predict prostate cancer outcome and response to treatment. 


\section{INTRODUCTION}

Prostate cancer is the most common cancer and the second leading cause of cancer death in men in the United States ${ }^{1}$. Despite the fact that most prostate cancers are diagnosed when they are still localized, as many as $30 \%$ of the cases will recur after an attempt at cure. Biochemically relapsed or metastatic prostate cancer is initially controlled by androgen suppression therapy. However, the cancer eventually becomes resistant to these maneuvers and frequently lethal. Much effort has been put into finding suitable predictive markers that could help distinguish those prostate cancer cases that will become more aggressive and will likely recur after treatment from tumors that are more indolent, in which a conservative management approach would be preferred to avoid over-treatment ${ }^{2}$.. Challenges exist in the management of advanced disease, where the clinical phenotype is heterogeneous, the ability to predict subsequent natural history is limited, and therapy choices are hampered by these knowledge gaps. Predictive biomarkers in this area are needed.

Epidemiologic evidence has linked prostate cancer occurrence to chronic inflammation causing factors such as infectious agents, chronic and persistent infections, pro-inflammatory, hormonal or dietary factors ${ }^{3,4}$. In some studies, chronic use of anti-inflammatory agents has been associated with a protective effect against prostate cancer ${ }^{5}$. Inflammatory factors may lead to resistance to therapy in advanced disease as well.

Elevated C-reactive protein [CRP], one of the most common and routinely measured markers of systemic inflammation, has been associated with the risk of developing specific cancers $^{6}$, such as colorectal and lung cancer ${ }^{7,8}$, and it has been associated with a poor prognosis for overall cancer as well as specifically for breast, lung, gastric, colorectal cancer, and renal cell carcinoma 9,10 .

Previous studies have reported that higher CRP levels are significantly associated with worse outcome in prostate cancer patients ${ }^{11,12}$, while others did not confirm the results ${ }^{13}$. The size of each individual study was not large enough to allow sufficient statistical power to assess any firm conclusion. A recently conducted meta-analysis ${ }^{14}$ suffers all the limitations of all metaanalyses, such as the poor comparability of published data across studies and the inability to adjust the results for possible confounders. We conducted a pooled analysis of individual data of studies on CRP and overall survival; individual data allows to conduct a sensitivity analysis to find the optimal CRP cut-off predicting survival. 


\section{METHODS}

Original research studies that evaluated prostate cancer survival in relation to CRP levels were identified by searching the National Library of Medicine and National Institutes of Health PubMed database and Embase. The search strategy included the following keyword search terms: "prostate cancer", "outcome", "CRP", "C reactive protein", and spanned from January 1990 to January 2014. Reference lists from all retrieved articles were also reviewed in search of additional eligible articles. Eligibility: Studies were considered eligible according to the following a priori criteria: (1) written in English; (2) availability of HR and 95\% Confidence Intervals (CI) according to CRP. Eight studies were identified; two of them were partially overlapping, and the most recent was used ${ }^{15,16}$. One study ${ }^{13}$ reported the $\mathrm{p}$ value for the lack of association between survival and CRP, but did not include the HR. The total eligible studies were therefore 6 . Corresponding authors of identified studies were contacted and invited to share individual data in order to conduct a pooled analysis of the association between CRP and prostate cancer outcome. Two data sets were received. (Table 1)

Statistical Analysis: In order to evaluate the quality of the data, hazard ratio for mortality in relationship to CRP cut-off values was extracted from each study. Preliminary to the pooled analysis, meta-analysis computations were performed using the "metan" command in Stata (Stata Version 10, StataCorp LP, College Station, TX) was used. The combined hazard ratio was calculated according to both a fixed and a random effects model; the Q statistics were used to test for heterogeneity between the studies included in the meta-analyses ${ }^{17}$. The $\mathrm{I}^{2}$ statistic was used as a confirmatory test for heterogeneity ${ }^{18}$, with $\mathrm{I}^{2}<25 \%, 25-50 \%$, and $>50 \%$ representing low, moderate and high degree of heterogeneity, respectively.

Pooled analysis: Individual data from two data sets were received and analyzed for survival in relation to CRP. Hazard ratios and $95 \%$ confidence intervals were calculated; multivariable Cox proportional hazards models provided estimates of overall survival, adjusted for age $(\leq>>71.9$ years), and available prognostic factors: PSA $(\leq />71.8 \mathrm{ng} / \mathrm{mL}), \mathrm{Hb}(\leq />12.5 \mathrm{~g} / \mathrm{dL})$, Alkaline Phosphatase $(\leq />113 \mathrm{U} / \mathrm{L})$. These cut off points represented the median value for the analyzed population. 
Analysis of the optimal CRP cut-off: The best cutoff of CRP was explored using X-title software

${ }^{19}$, which is based on log-rank test statistics, where categorical (high/ medium/low or high/low) CRP variables were generated, using the individual data from the available two data sets, and used as the predictor in Cox proportional hazards models. The best cutoff was defined as the point with the most significant split (i.e. the maximum $\chi^{2}$ ) among the survival distributions of those with different categorical CRP levels. The statistical significance was indicated by uncorrected and cross-validation p-values. In addition, an adjusted p-value based on the MillerSiegmund method ${ }^{20}$ was obtained for high/low models.

\section{RESULTS}

There were 6 studies available, and 699 prostate cancer patients included. Two studies were conducted in Japan, 3 in US, 1 in UK. Five studies included advanced prostate cancer, one ${ }^{15}$ was conducted on localized prostate cancer cases. The CRP cut-off value used in the original analysis varied from $>5 \mathrm{mg} / \mathrm{l}^{12,21,22}$, to $10 \mathrm{mg} / \mathrm{l}^{15}$, to $15 \mathrm{mg} / \mathrm{l}^{23}$.

All the studies showed a statistically significant association between CRP and prostate cancer survival. The overall meta- hazard ratio (HR $\mathrm{HR}_{\text {mta }}$ ) was 1.83 (95\% CI: 1.51-2.21), without evidence of heterogeneity among studies ( $\mathrm{Q}$ statistics: $2.7, \mathrm{p}=0.6$; Figure 1 ). The restricted analysis to those studies that used a common cut off point of $5 \mathrm{mg} / 1$ [3 studies $\left.{ }^{12,21,22}\right]$ produced a HR meta of 1.78 (1.39-2.27), with no evidence of heterogeneity.

Pooled analysis: There were individual data for 235 patients ( 2 of the 6 studies included in the meta-analysis above ${ }^{12,21}$ ); their description is reported in table 2. The crude $\mathrm{HR}_{\text {pooled }}$ for $\mathrm{CRP}<5$ vs $\geq 5 \mathrm{mg} / 1$ was 1.68 (1.23-2.29), the adjusted HRpooled was 1.44 (1.02-20.4) (Figure 1).

Optimal CRP cut-off: Using the pooled analysis, the best CPR cutoff, when the option of one cut-off comparing high versus low levels was chosen, was $12 \mathrm{mg} / 1$ (corrected $\mathrm{p}$-value $=0.01$; uncorrected $p$-value $=0.0005)$. The $\mathrm{HR}_{\text {pooled }}$ for $\mathrm{CRP}<12 \mathrm{vs} \geq 12 \mathrm{mg} / \mathrm{l}$ was $1.78(1.29-2.47)$ and 1.53 (1.01-2.32) for the crude and adjusted models, respectively (Figure 2).

\section{DISCUSSION}


The present pooled analysis confirms previous findings of an association between CRP levels and overall survival. The meta-analysis of published studies suggests a statistically significant $83 \%$ increased risk of dying for those patients with high CRP in comparison with those with low CRP. As always with meta-analyses, some limitations apply; the comparison across included studies show that the cut-off for CRP vary, with the most commonly used cut-off being $5 \mathrm{mg} / \mathrm{L}$. A sub-set analysis was conducted on the studies relying on the same CRP cut-off, confirming the results of the larger analysis. Another limitation is the heterogeneity of the populations of prostate cancer patients included in each study. Some studies are conducted on metastatic patients and others on localized prostate cancer. In addition, the summary estimates cannot be adjusted for possible confounders of the association, such as, for example other prognostic factors, or age.

In an attempt to overcome the limitations, a pooled analysis of individual data was conducted, including 235 patients. The pooled analysis indicates that a CRP value over $5 \mathrm{mg} / \mathrm{l}$ at baseline is a significant predictor of survival, even after adjusting the analyses for other prognostic factors. It should be noted that the patients in this pooled analysis were similar in that they had mCRPC, many of whom received chemotherapy, but all were treated in an era before enzalutamide, abiraterone, cabazitaxel, sipuleucel-T and radium-223, the five new therapies for mCRPC approved since 2010.

This analysis has some weaknesses worth mentioning. Only 2 of the 6 datasets were available for the pooled analysis of individual data. Although the authors of the other publications were contacted several times and asked to send their data, they never responded. The data from the ASCENT study are not available since the company that ran the study is no longer existent. We were able to perform a more rudimentary analysis with all 6 studies using group, rather than individual adjusted statistics, and obtained similar results as the pooled analysis, specifically a statistically significant negative impact of elevated CRP on survival. However, the similarities and differences between the data submitted for the pooled analysis versus the data that was not submitted is worth mentioning. The two datasets received were homogeneous in that they were both from North America, and included men with mCRPC treated on a clinical trial. Two of the four datasets not submitted included patients treated in Japan ${ }^{22,23}$, two did not specify castration-resistant prostate cancer ${ }^{15,23}$. Even without the four other datasets, this is still the largest patient group to be analyzed for the prognostic value of 
CRP in prostate cancer. As always, there are limitations to this analysis: one is the lack of cancer-specific details. For example, Gleason grade values on half of the patients in the pooled analysis, and this prevented the inclusion of the Gleason score in the multivariate analysis. Gleason is an important factor in prognosis, but not every reported nomogram includes it. The Armstrong nomogram (TAX327) and Halabi nomogram (CLGB-B) used it, whereas the Armstrong risk groups, Smaletz nomogram (MSKCC) and PCWG-2 clinical subtypes do not use it. Furthermore, we did not have access to full staging, for example number of metastases, and this information could affect CRP values; full treatment details of the primary cancer (adjuvant radiation) was also incomplete. Other factors influencing CRP values, for example the presence of comorbidities, was not available in this sample of patients, Finally, the methods for CRP measurement in the Pond paper were not known at the time of this analysis.

The biological reasons for the association between CRP and survival in prostate cancer are unclear. From the available data sets it is impossible to ascertain, for example, if CRP is increased due to the presence of cancer, or because of the immune state of the host, independent of other conditions or other chronic co-morbidities such as infection or cardiovascular disease, as CRP is also an independent predictor of cardiovascular mortality ${ }^{24}$. It has been pointed out that CRP levels also increase as a response to chronic inflammation to ionizing radiation used for treating localized prostate cancer ${ }^{25}$. CRP also represents a regulating factor on tumor growth and proliferation ${ }^{26}$, thus it could be contributing to cancer aggressiveness.

Another aspect to consider is that the association between CRP and cancer survival is not specific to prostate cancer, but has been seen in other cancers as well ${ }^{9,27-30}$. It is possible that CRP simply reflects an enhanced immune response elicited by the tumor, and such response is more intense when local tissue damage and inflammation are high, as happens with more aggressive cancer sub-types. Interestingly, other markers of a systemic inflammatory and immune state have also appeared prognostic in prostate cancer of different stages, as well as other malignancies, e.g. the neutrophil by lymphocyte ratio ${ }^{31}$.

Taking advantage of the data set available for analysis, we conducted a sensitivity analysis, to understand what the best CRP cut-off value is that could predict prostate cancer outcome. This analysis suggests that a value of $12 \mathrm{mg} / \mathrm{l}$ should be considered in future analyses. 
The observation that a marker of inflammation, routinely measured as part of the baseline lab work in many clinical settings, could be used as a prognostic biomarker requires further investigation, including possibly interventions to modify CRP levels during cancer follow-up in an attempt to prevent recurrence and ultimately death. Another future path of research should be the study of local prostate tissue patterns among those patients with high CRP, and the comparison with prostate tissues from patients with low CRP, in an attempt to understand if CRP is reflecting a specific aggressive micro-environment.

In conclusion, we report here the results of a pooled analysis showing a statistically significant association between elevated CRP levels and survival. Since CRP is an affordable and readily available assay, it may hold promise in improving prognostication and potentially to predict the activity of specific therapeutic agents. Indeed, the prospective evaluation of CRP as a prognostic marker is ongoing in the AFFINITY phase III trial, which is investigating the impact of combining custirsen with cabazitaxel in metastatic castration resistant prostate cancer. The test can be introduced in clinical routine evaluation only when prospective data will be available showing that CRP provides important information for prognosis.

Funding source: this work was partially supported by DOD PC120302 and NIH U01 $\mathrm{OH} 010396$ to ET

\section{THE AUTHORS DECLARE NO CONFLICT OF INTEREST}




\section{REFERENCES}

1. Siegel R, Ma J, Zou Z, Jemal A. Cancer statistics, 2014. CA: A Cancer Journal for Clinicians $2014 ; 64: 9-29$

2. Briganti A, Suardi N, Gallina A, Abdollah F, Novara G, Ficarra V, Montorsi F. Predicting the risk of bone metastasis in prostate cancer. Cancer Treat Rev. 2014; 40(1):3-11

3. De Marzo AM, Platz EA, Sutcliffe S, Xu J, Grönberg H, Drake CG, Nakai Y, Isaacs WB, Nelson WG. Inflammation in prostate carcinogenesis. Nature Reviews Cancer 2007; 7: 256-69.

4. Coussens LM, Werb Z. Inflammation and cancer. Nature 2002; 420: 860-7.

5. Mahmud S, Franco E, Aprikian A. Prostate cancer and use of nonsteroidal anti-inflammatory drugs: systematic review and meta-analysis. Br J Cancer 2004; 90: 93-9

6. Guo YZ1, Pan L, Du CJ, Ren DQ, Xie XM. Association between C-reactive protein and risk of cancer: a meta-analysis of prospective cohort studies. Asian Pac J Cancer Prev. 2013; 14 (1): 243-8.

7. Heikkila K, Ebrahim S, Lawlor DA. A systematic review of the association between circulating concentrations of $\mathrm{C}$ reactive protein and cancer. J Epidemiol Comm Health 2007; 61: 824-33.

8. dos Santos Silva I, De Stavola BL, Pizzi C, Meade TW. (2010) Circulating levels of coagulation and inflammation markers and cancer risks: individual participant analysis of data from three long-term cohorts. Intl J Epidemiol 2010; 39: 699-709.

9. Roxburgh CS, McMillan DC. Role of systemic inflammatory response in predicting survival in patients with primary operable cancer. Future Oncology 2010; 6: 149-63.

10. Wu Y, Fu X, Zhu X, He X, Zou C, Han Y,et al. Prognostic role of systemic inflammatory response in renal cell carcinoma: a systematic review and meta-analysis. J Cancer Res Clin Oncol 2011; 137: 887-96. 
11. Beer TM, Lalani AS, Lee S, Mori M, Eilers KM, Curd JG, Henner WD, Ryan CW, Venner P, Ruether JD, Chi KN. C-reactive protein as a prognostic marker for men with androgenindependent prostate cancer: results from the ASCENT trial. Cancer 2008; 112:2377-2383. 12. Prins RC, Rademacher BL, Mongoue-Tchokote S, Alumkal JJ, Graff JN, Eilers KM, Beer TM. C-reactive protein as an adverse prognostic marker for men with castration-resistant prostate cancer (CRPC): Confirmatory results. Urol Oncology: Seminars and Original Investigations $2012 ; 30$ : 33-37

13. Elsberger B, Lankston L, McMillan DC, Underwood MA, Edwards J. Presence of tumoural C-reactive protein correlates with progressive prostate cancer. Prostate Cancer Prostatic Dis. 2011;14(2):122-8.

14. Liu ZQ, Chu L, Fang JM, Zhang X, Zhao HX, Chen YJ, Xu Q Prognostic role of C-reactive protein in prostate cancer: a systematic review and meta-analysis. Asian J Androl. 2014; 16(3):467-71.

15. McArdle PA, Qayyum T, McMillan DC. Systemic inflammatory response and survival in patients with localised prostate cancer: 10-year follow-up. Urol Int. 2010; 84(4):430-5. 16. McArdle PA, Mir K, Almushatat AS, Wallace AM, Underwood MA, McMillan DC. Systemic inflammatory response, prostate-specific antigen and survival in patients with metastatic prostate cancer. Urol Int. 2006; 77(2):127-9.

17. Higgins JP, Thompson SG. Quantifying heterogeneity in a meta-analysis. Stat.Med 2002; 21:1539-58.

18. Ioannidis JPA, Patsopoulos NA, Evangelou E. Uncertainty in heterogeneity estimates in meta-analyses. BMJ 2007; 335:914-6. 
19. Camp RL, Dolled-Filhart M, Rimm DL. X-Tile: A New Bio-Informatics Tool for Biomarker Assessment and Outcome-Based Cut-Point Optimization. Clin Cancer Res 2004; 10:7252-7259 20. Miller R, Siegmund, D. Maximally selected chi square statistics. Biometrics 1982; 38:10111016.

21. Pond GR, Armstrong AJ, Wood BA, Leopold L, Galsky MS, Sonpavde G. Ability of Creactive protein to complement multiple prognostic classifiers in men with metastatic castration resistant prostate cancer receiving docetaxel-based chemotherapy. B J U Int 2012; 110: 461-4 68 22. Ito M, Saito K, Yasuda Y, Sukegawa G, Kubo Y, Numao N, Kitsukawa S, Urakami S, Yuasa T, Yamamoto S, Yonese J, Fukui I. Prognostic impact of C-reactive protein for determining overall survival of patients with castration-resistant prostate cancer treated with docetaxel. Urology 2011; 78(5):1131-5

23. Nakashima J, Kikuchi E, Miyajima A, Nakagawa K, Oya M, Ohigashi T, Murai M. Simple Stratification of Survival Using Bone Scan and Serum C-Reactive Protein in Prostate Cancer Patients with Metastases. Urol Int 2008; 80:129-133

24. Albert MA, Danielson E, Rifai N, Ridker PM. Effect of statin therapy on C-reactive protein levels: the pravastatin inflammation=CRP evaluation (PRINCE): a randomized trial and cohort study. JAMA 2001; 286:64-70.

25. Hall W A, Nickleach DC, Master VA, Prabhu RS, Rossi PJ, Godette K, Cooper S, Jani AB. The Association Between C-Reactive Protein (CRP) Level and Biochemical Failure-Free Survival in Patients After Radiation Therapy for Nonmetastatic Adenocarcinoma of the Prostate. Cancer $2013 ; 119: 3272-9$ 
26. Halabi S, Small EJ, Kantoff PW, Kattan MW, Kaplan EB, Dawson NA, Levine EG, Blumenstein BA, Vogelzang NJ. Prognostic model for predictingsurvival in men with hormonerefractory metastatic prostate cancer. J Clin Oncol 2003; 21:1232-7.

27. McMillan DC, Canna K, McArdle CS. A systemic inflammatory response predicts survival following curative resection for colorectal cancer. Br J Surg 2003; 90: 215-219.

28. Jamieson NB, Glen P, McMillan DC, McKay CJ, Foulis AK, Carter R, Imrie CW. Systemic inflammatory response predicts outcome in patients undergoing resection for ductal adenocarcinoma head of pancreas. Br J Cancer 2005; 92: 21-23.

29. Crumley ABC, McMillan DC, McKernan M, Going JR, Stuart RC. The relationship between the pre-operative systemic inflammatory response and cancer specific survival in patients undergoing potentially curative resection for gastro-oesophageal cancer. Br J Cancer 2006; 94 : $1568-1571$.

30. Yoshida S, Saito K, Koga F, Yokoyama M, Kageyama Y, Masuda H, Kobayashi T, Kawakami S, Kihara K. C-reactive protein level predicts prognosis in patients with muscleinvasive bladder cancer treated with chemoradiotherapy. BJU Int 2008; 101: 978-981. 31. Keizman D, Gottfried M, Ish-Shalom M, Maimon N, Peer A, Neumann A. Pretreatment neutrophil-to-lymphocyte ratio in metastatic castration-resistant prostate cancer patients treated with ketoconazole: association with outcome and predictive nomogram. The Oncologist 2012; 17:1508-14. 
Figure 1 - Pooled estimates of overall survival of prostate cancer patients according to CRP values $(\mathrm{N}=$ 235)

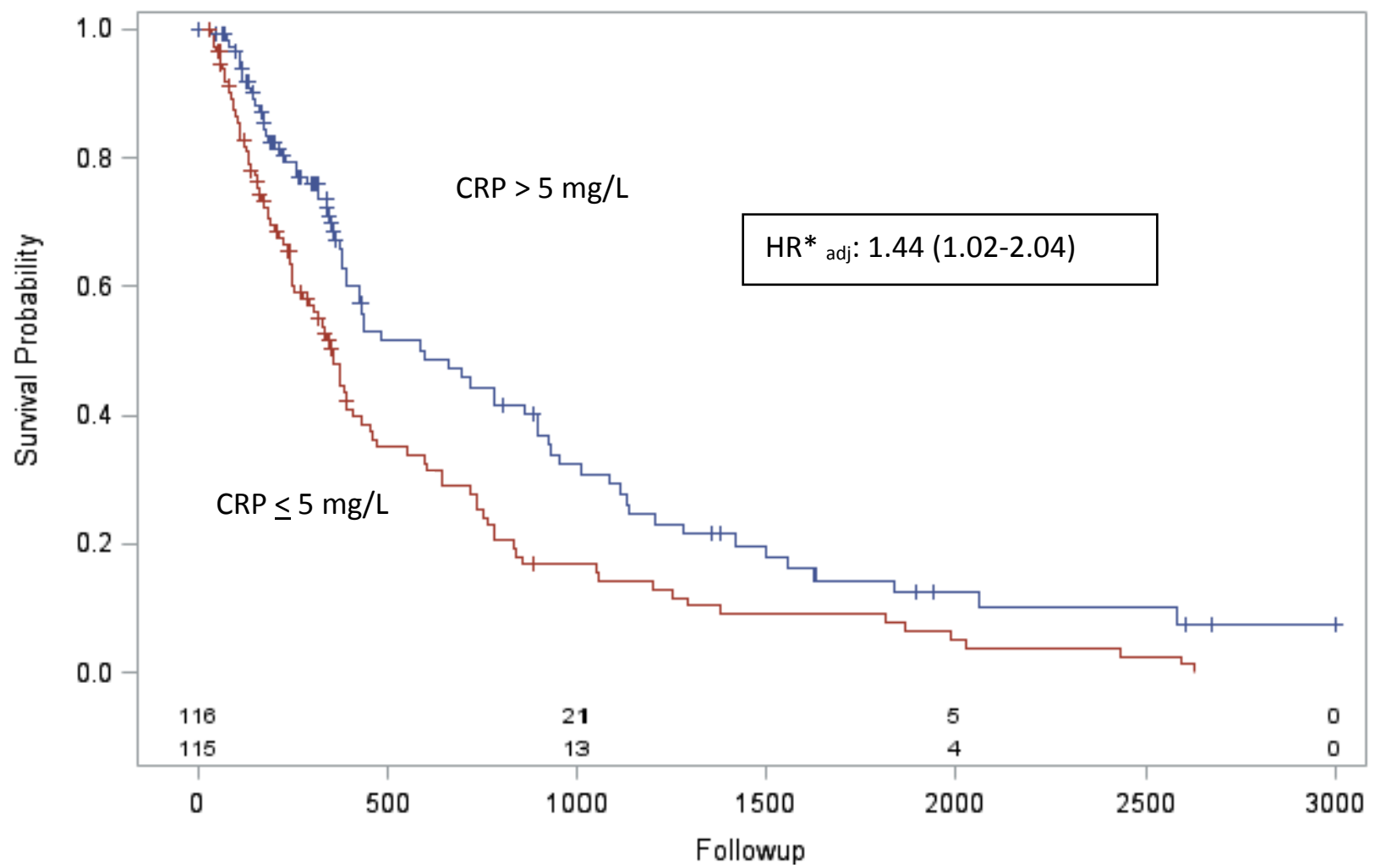

*adjusted for age $(\leq />71.9$ years), PSA $(\leq />71.8 \mathrm{ng} / \mathrm{mL}), \mathrm{Hb}(\leq />12.5 \mathrm{~g} / \mathrm{dL})$, Alkaline Phosphatase $(\leq />$ $113 \mathrm{U} / \mathrm{L})$ 
Figure 2 - survival estimate using the best cut-off point of $12 \mathrm{mg} / \mathrm{L}$

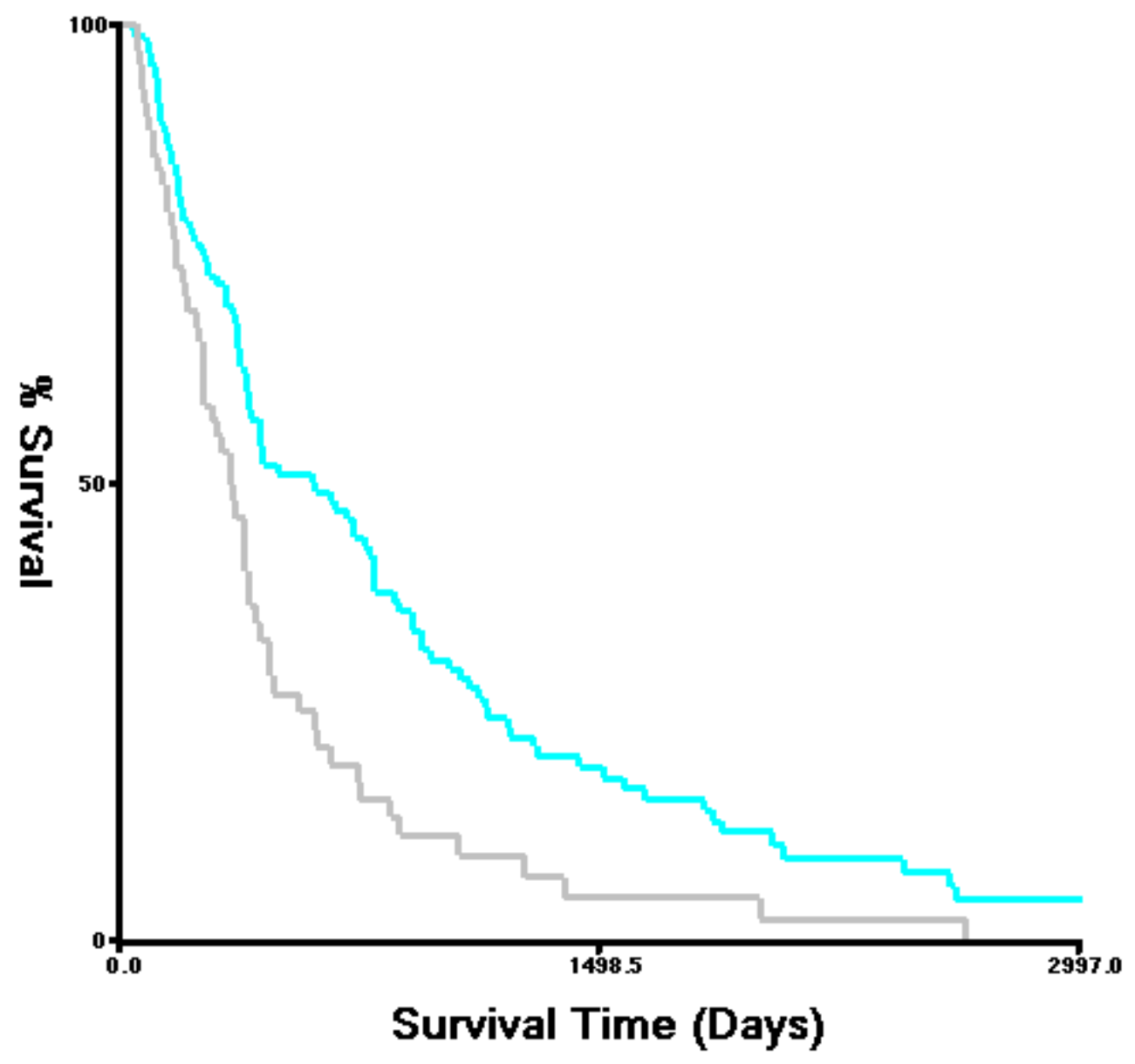


Table 1 - summary of studies on the association between CRP and prostate cancer survival

\begin{tabular}{|c|c|c|c|c|c|c|}
\hline AUTHOR, REF & COUNTRY & $\mathbf{N}$ & Population & CRP cut off & HR for survival & Estimate \\
\hline Ito 22 & Japan & 80 & $\begin{array}{l}\text { CRPC, at least } 88 \% \\
\text { metastatic }\end{array}$ & $\geq 5.0 \mathrm{mg} / \mathrm{l}$ & $1.95(1.33-2.96)$ & Crude \\
\hline Nakashima ${ }^{23}$ & Japan & 126 & Metastatic PC & $\leq 15 \mathrm{mg} / \mathrm{l}$ & $1.88(1.03-3.45)$ & Adjusted \\
\hline Beer, $2008^{11}$ & US & 160 & $\begin{array}{l}\text { mCRPC treated with } \\
\text { docetaxel-based } \\
\text { chemotherapy }\end{array}$ & $8 \mathrm{mg} / \mathrm{L}$ & $2.96(1.52-5.77)$ & Adjusted \\
\hline Pond ${ }^{21} *$ & US & 116 & $\begin{array}{l}\text { mCRPC treated with } \\
\text { docetaxel-based } \\
\text { therapy; } \underline{\mathrm{CRP} \text { prior to }} \\
\text { treatment }\end{array}$ & \multirow[t]{2}{*}{$5.0 \mathrm{mg} / \mathrm{l}$} & \multirow[t]{2}{*}{$1.68(1.23-2.29)$} & \multirow[t]{2}{*}{ Crude } \\
\hline Prins, $2012^{12} *$ & US & 119 & $\begin{array}{l}\text { CRPC }(90.8 \% \\
\text { mCRPC); various } \\
\text { treatments, CRP prior } \\
\text { to treatment }\end{array}$ & & & \\
\hline Mc Ardle ${ }^{15}$ & UK & 98 & Localized PC & $\leq 3 ; 3-10 ;>10$ & $1.63(1.08-2.45)$ & Crude \\
\hline \multirow[t]{2}{*}{ TOTAL } & & 699 & & & & \\
\hline & & & & & $\begin{array}{l}\text { Meta HR (95\% } \\
\mathrm{Cl})^{\wedge}\end{array}$ & \\
\hline $\begin{array}{l}\text { Fixed model } \\
\text { Random model }\end{array}$ & & & & & $\begin{array}{l}1.83(1.51-2.21) \\
1.83(1.51-2.21)\end{array}$ & \\
\hline
\end{tabular}

*individual data received

$\mathrm{PC}=$ Prostate Cancer

$\mathrm{mCRPC}=$ metastatic, castration resistant prostate cancer

${ }^{\wedge} Q$ statistics for heterogeneity: 2.7 ( $p$ value: 0.6$) ; I^{2}:<5 \%$ 
Table 2 - description of patients included in the pooled analysis $(\mathrm{N}=235)$

\begin{tabular}{|l|l|l|}
\hline VARIABLE & Mean \pm SD (range) & $\mathrm{N}(\%)$ \\
\hline Age (years) & & \\
\hline CRP (mg/L) & $71.3 \pm 8.7(45.8-91.5)$ & \\
\hline Hemoglobin (g/dL) & $19.4 \pm 39.1(0.3-311)$ & \\
\hline Alkaline Phosphatase (U/L) & $12.4 \pm 1.6(7.4-16.7)$ & \\
\hline PSA (ng/mL) & $194.3 \pm 223.9(33-1436)$ & \\
\hline Length of follow -up (days) & $191.2 \pm 327.3(0.8-2112.6)$ & \\
\hline Dead at follow -up & $510 \pm 578.6(2-2997)$ & \\
\hline ECOG* & & $166(70.6)$ \\
\hline 0 & & \\
\hline 1 & & $102(43.4)$ \\
\hline$\geq 2$ & & $100(42.6)$ \\
\hline
\end{tabular}

*= Eastern Cooperative Oncology Group 